tify primary and secondary protein targets of bioactive compounds, including those whose mechanism of action is unknown. Proteindrug complexes are pulled out of cells using a tag present on the drug. The proteins are then processed on a 'proteomics reactor', developed at the company, that simulates the physiological protein concentrations found in cells, making it more likely that the protein complexed with the drug can be chemically or biochemically processed and analysed by mass spectrometry.

A tough challenge for proteomics is the detection of integral membrane proteins which include important drug targets. By focusing on quantitative proteomic profiling of plasma-membrane proteins, drugdiscovery company Caprion in Montreal, Canada, hopes to home in on the $1 \%$ of proteins in the cell that are likely to be the most relevant drug targets. Its proprietary CellCarta platform is an integrated and automated suite of technologies for fractionation of human tissue samples and plasma, quantitative analysis and directed mass spectrometry-based identification of protein targets.

\section{Inside the intact cell}

"The big issue is, what is physiological? What is going on in vivo? How do proteins come together and interact in intact cells?" says Sam Hanash of the University of Michigan Medical School, Ann Arbor, and president of the Human Proteome Organization. This international effort in proteomics focuses on organ systems and biological fluids relevant to diseases. "Some exciting technologies are becoming available that may not be necessarily high-throughput but are more physiological." He cites methods that allow visualization of protein modifications in living cells, such as genetically encoded fluorescent indicators that detect protein-phosphorylation in signal transduction, for example.

A well-established technique for detecting protein-protein interactions is yeast two-hybrid analysis. "It's very powerful and you generate many interactions. But it is the first level of proteome mining; you then require more detailed follow-up and validation," says David Litman, senior vicepresident of R\&D and chief technology officer at BD Biosciences in San Jose, California, which makes the BD Matchmaker system for yeast two-hybrid analysis.

"A lot of proteomics is done in bulk, people taking cells and cracking them open to see what proteins are in there," says Litman. "But after scanning the whole proteome, one would want to analyse smaller sets of proteins." Antibodies to detect phosphorylated proteins are being commercialized by BD Biosiences as multiplexed immunoassays to interrogate intracellular pathways using flow cytometry. The new BD FACSArray bioanalyser accepts 96-well plates for high-throughput cellular analysis or multiplexed protein analysis using cytometric beads. A microtitre plate can be run in less than 35 minutes, detecting up to 1,000 events per well and reporting up to four fluorescent and two optical scattering parameters. With this benchtop system, researchers can study protein interactions within a given pathway without having to separate cell populations before running the assay. Reagents to detect kinases, phosphorylated proteins and other activated protein states are available, and $\mathrm{BD}$ Biosciences is developing preconfigured kits.

Protein-capture microarrays are limited as they stand, partly because of nonspecific binding by the capture agents, says James Wang, chief technology officer at Hypromatrix in Worcester, Massachusetts. "Additional protein microarray formats are needed," he says. To increase specificity and expand array applications, Hypromatrix has developed the Staining AntibodyArray to simultaneously detect and localize large numbers of cellular antigens in intact cells. Detector antibodies are arrayed on one support, while the cells are attached to a second. When the two surfaces are apposed, antibodies dissociate from the array and bind to cell-surface antigens. After the supports are separated, secondary antibodies are used to profile the antigens revealed and their cellular location.

The proteomics boom continues. And although cutting-edge proteome analysis is expensive, the growing array of proteomics tools promises to supply researchers with a method suited to every experimental need. Lisa Melton is science writer at the Novartis Foundation, London, UK.

\title{
BREAKING DOWN THE PROBLEM
}

A number of companies have developed peptide arrays for detecting protein interactions, such as the interaction of protein kinases with their substrates. Using high-throughput peptide synthesis, they manufacture an entire potential protein target as overlapping peptides and array them on a chip or a membrane. Pepscan Systems in Lelystad, the Netherlands, for example, offers the pre-arrayed PepChip, with 1,200 peptide substrates for protein kinase identification and assay, while New England Peptide in Gardner,

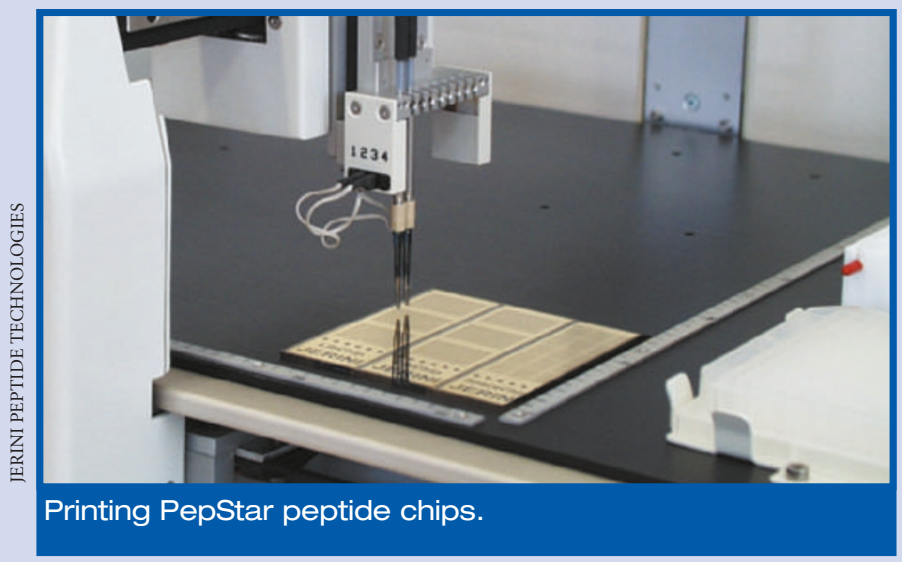

Massachusetts, has adopted the 96-well microplate format for its custom peptide arrays, with a different synthetic peptide in each well.

"The advantage is that smaller peptides are less dependent than proteins on secondary structure for their biological function," explains Holger Wenschuh of Jerini Peptide Technologies in Berlin, Germany. Jerini identifies peptide substrates for customers' orphan kinases, and for other categories of protein-modifying enzymes, such as proteases and phosphatases, using its PepStar microarray platform, in which up to 20,000 peptide sequences are synthesized on a membrane and then transferred to the complementary chip. The customer sends in their enzyme, Jerini systematically trawls the available literature and protein databases, such as Swiss-Prot and Phosphobase for potential substrates, sets up arrays, does the assay experiments and delivers the peptide substrate information, often in less than a week. Jerini also offers a ready-to-use PhosphoSite-Detector microarray kit to detect potential phosphorylation sites in kinase substrates. The readout is a phosphate-transfer event, which can be detected by autoradiography or by a phosphotyrosine-specific antibody.

Sigma's Michael Hadjisavas sees custom peptide arrays as "an area that is poised to explode". Custom peptide arrays using Sigma's PEPscreen platform are used to map epitopes, protein-protein interactions or protein-ligand interactions. "Since roughly half of all biotherapeutics are antibodies," says Hadjisavas, "it is important to demonstrate the specific peptide sequence that is being recognized in the target protein." 